\title{
Transient electroluminescence measurements on organic heterolayer light emitting diodes
}

\author{
Anton G. Mückl *, Stefan Berleb, Wolfgang Brütting, Markus Schwoerer \\ University of Bayreuth, Experimental Physics II, 95440 Bayreuth, Germany
}

\begin{abstract}
We have investigated the field dependence of the electron and hole drift mobility in tris-(8-hydroxyquinoline) aluminium (Alq ${ }_{3}$ ) in organic multi layer light emitting diodes (LEDs) using transient electroluminescence measurements. The electron transport in thin films of $\mathrm{Alq}_{3}$ can be understood in terms of hopping processes. We obtain a full set of parameters for a modified Poole-Frenkel equation describing the temperature and field dependence of the electron drift mobility. For the measurement of the hole drift mobility, a special device structure was designed. We were able to determine the hole drift mobility in $\mathrm{Alq}_{3}$ and found good agreement with the data obtained from previous time-of-flight (TOF) measurements.
\end{abstract}

Keywords: Electroluminescence measurements; $\mathrm{Alq}_{3}$; Light emitting diodes

\section{Introduction}

Since the first efficient devices were demonstrated in 1987 [1], organic light emitting diodes (LEDs) gained increasing interest due to their possible application in displays. Considerable efforts were made to improve the performance of organic LEDs. Even though many new substances were developed and investigated tris-(8-hydroxyquinoline) aluminium $\left(\mathrm{Alq}_{3}\right)$, as used in the so-called Kodak structure, is still one of the most favored electron transports and/or light emitting materials. Following early time-of-flight (TOF) measurements by Kepler et al. [2] $\mathrm{Alq}_{3}$ is widely regarded as a purely electron conduction material with negligible hole drift mobility. On the other hand, TOF experiments on multi layer structures with $\mathrm{Alq}_{3}$ films indicate that the hole drift mobility in $\mathrm{Alq}_{3}$ may be comparable to the values for electrons [3]. Therefore, it is not clear whether the hole transport in $\mathrm{Alq}_{3}$ thin films can be neglected as done in some cases interpreting experimental data.

We went further into the question if this assumption is legitimate even at high electric fields. To the best of our knowledge, there are still scarce data on charge carrier mobility in $\mathrm{Alq}_{3}$ thin films in the range of electric fields where organic LEDs are typically operated [4]. In this

\footnotetext{
${ }^{*}$ Corresponding author.

E-mail address: anton.mueckl@uni-bayreuth.de (A.G. Mückl).
}

work, we present results of time resolved electroluminescence (EL) measurements on single hetero structure (SHS) organic LEDs based on the aromatic triphenyldiamine (TPD) and $\mathrm{Alq}_{3}$ at various temperatures. Using a modified Poole-Frenkel law, we obtain a full set of parameters for the description of the temperature and field dependence of electron drift mobility in $\mathrm{Alq}_{3}$.

It will be further shown that the transient behavior of electroluminescence upon application of a rectangular pulse to a double hetero structure (DHS) device is determined by the hole drift mobility in $\mathrm{Alq}_{3}$. We quantify the dependence of the hole drift mobility in $\mathrm{Alq}_{3}$ on the electric field.

\section{Experimental details}

The temperature dependent measurements of the electron drift mobility in $\mathrm{Alq}_{3}$ were performed on a TPD/ $\mathrm{Alq}_{3}$ SHS device as shown in Fig. 1a. The sample was mounted in a liquid nitrogen cryostat. After achieving the desired temperature, single rectangular voltage pulses (pulse width $1 \mathrm{~ms}$ ) were applied to the device using a pulse generator. The voltage pulse and electroluminescence signal obtained from a photomultiplier were sampled by a digital storage oscilloscope.

The hole drift mobility in $\mathrm{Alq}_{3}$ was measured using DHS devices (see Fig. 1b) consisting of two TPD/Alq 3 


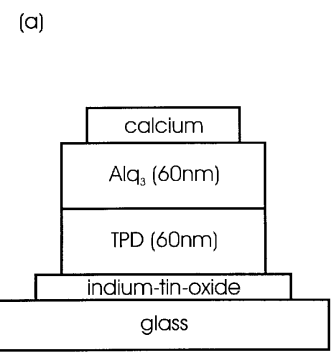

single hetero structure (SHS)

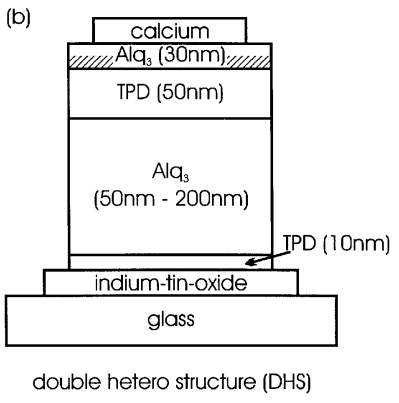

Fig. 1. (a) SHS: device used for transient measurements of electron drift mobility in $\mathrm{Alq}_{3}$. (b) DHS: device used for transient measurements of hole drift mobility in $\mathrm{Alq}_{3}$. The upper $\mathrm{Alq}_{3}$ layer is partially doped with $2 \%$ DCM. The doped region is indicated by the hatching.

double layers. To verify that the delay time of the electroluminescence signal is determined by the hole transit time in the $\mathrm{Alq}_{3}$ layer sandwiched between the two TPD layers, samples with three different layer thicknesses $d=50,100$, $200 \mathrm{~nm}$ were investigated. The upper $\mathrm{Alq}_{3}$ layer was partially doped with $2 \%$ DCM laser dye to identify this as emission layer. To determine the delay time, either single pulse measurements or an average over 10 to 40 pulses at a repetition frequency of 0.3 to $0.05 \mathrm{~Hz}$ were taken depending on the signal obtained from the photomultiplier. These measurements were performed at room temperature.

\section{Electron mobility in $\mathbf{A l q}_{3}$}

A simple model of transient electroluminescence assumes that holes and electrons are injected simultaneously at the electrodes when a rectangular pulse is applied to the device (the possibility of delayed injection of one carrier species due to finite injection barriers [5] will not be discussed here). The velocity of the charge carriers moving through the organic layer is determined by the mobility and the electric field. Electroluminescence first occurs when the leading edge of the carrier packages meet. At a given electrical field $F$ and layer thickness $d$, the delay time $t_{\mathrm{d}}$ depends on the mobility as

$t_{\mathrm{d}}=d / \mu F$.

If the mobilities of both charge carrier types are very different, instead of the ambipolar mobility $\mu=\mu_{\mathrm{e}}+\mu_{\mathrm{h}}$ the higher drift mobility determines the delay time.

Measuring the time lag between the leading edge of an applied rectangular pulse with amplitude $V$ and the onset of electroluminescence (see Fig. 2) one can calculate the mobility as

$\mu=d /\left(t_{\mathrm{d}} \cdot F\right)$ with $F=\left(V-V_{\mathrm{bi}}\right) / d$.

At low voltages, the built-in field $V_{\mathrm{bi}}$ has to be considered as the electrode materials used in organic LEDs have

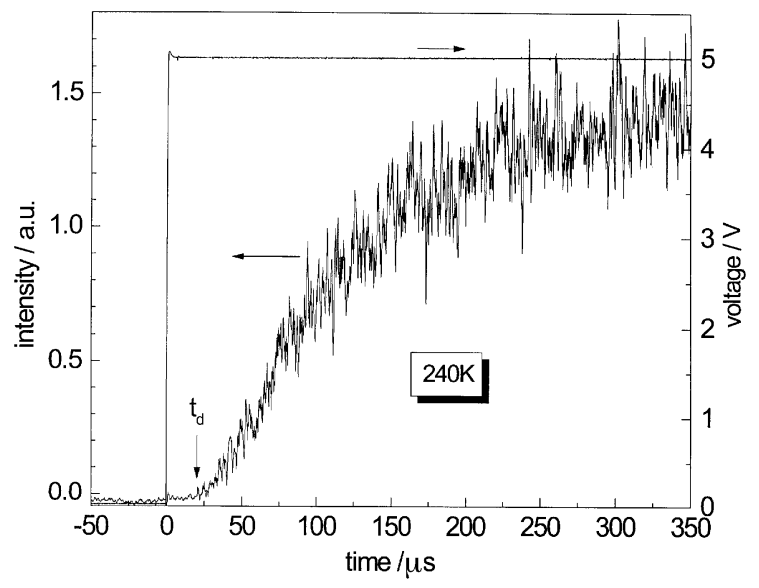

Fig. 2. Delay of electroluminescence intensity after application of a single voltage pulse with an amplitude of $5 \mathrm{~V}$ at $240 \mathrm{~K}$. The delay time $t_{\mathrm{d}}$ is approximately $21 \mu \mathrm{s}$.

different work functions. For the used ITO and $\mathrm{Ca}$ electrodes we have determined $V_{\mathrm{bi}} \approx 1.8-2.0 \mathrm{~V}$.

As the mobility of holes in TPD is higher by approximately three orders of magnitude than the mobility of electrons in $\mathrm{Alq}_{3}\left(\mu_{\mathrm{h}, \mathrm{TPD}} \gg \mu_{\mathrm{e}, \mathrm{Alq}_{3}}\right)$ most of the applied voltage drops at the $\mathrm{Alq}_{3}$ layer. For this reason, only the thickness of the $\mathrm{Alq}_{3}$ layer has to be considered in the calculation of the electrical field. Thus, the delay of electroluminescence is determined by the transit time of electrons in the $\mathrm{Alq}_{3}$ layer as holes and electrons recombine in the $\mathrm{Alq}_{3}$ layer near the $\mathrm{TPD} / \mathrm{Alq}_{3}$ interface due to the higher mobility of electrons as compared to holes in $\mathrm{Alq}_{3}$ $\left(\mu_{\mathrm{e}, \mathrm{Alq}_{3}} \gg \mu_{\mathrm{h}, \mathrm{Alq}_{3}}\right)$.

We have measured the delay times in transient electroluminescence measurements for different applied voltages from 2 to $14 \mathrm{~V}$ and in the temperature range between 300 $\mathrm{K}$ and $120 \mathrm{~K}$. The obtained mobilities according to equation (2) are plotted vs. the square root of the electric field in Fig. 3.

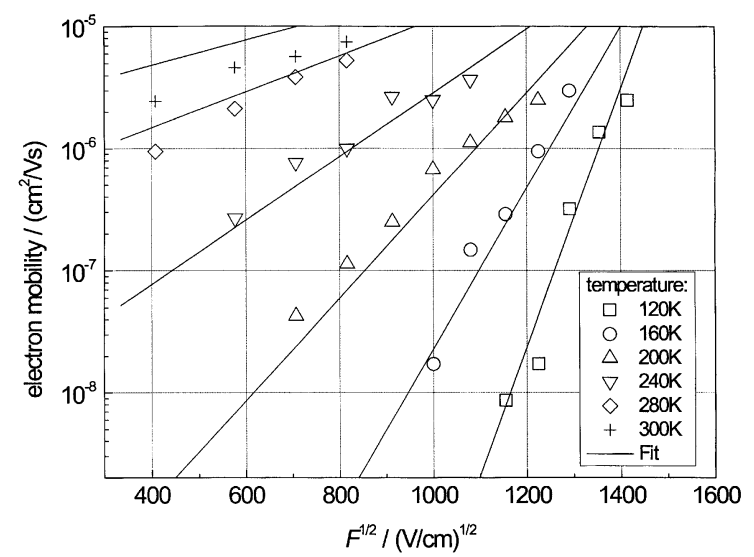

Fig. 3. Temperature and field dependence of electron drift mobility in $\mathrm{Alq}_{3}$. 
The drift mobility of electrons in $\mathrm{Alq}_{3}$ can be described in terms of hopping processes following a modified Poole-Frenkel law [6,7] as

$\mu=\mu_{0} \exp \left(-\frac{\Delta E-\beta_{\mathrm{PF}} \sqrt{F}}{k_{\mathrm{B}} T_{\text {eff }}}\right), \frac{1}{T_{\text {eff }}}=\frac{1}{T}-\frac{1}{T_{0}}$

where $E$ is the activation energy at zero electric field, $T$ the temperature, $T_{0}$ an empirical parameter and $k_{\mathrm{B}}$ Boltzmann's constant. The Poole-Frenkel factor is given by

$\beta_{\mathrm{PF}}=\sqrt{q^{3} /\left(\pi \varepsilon_{\mathrm{r}} \varepsilon_{0}\right)}$

where $q$ is the elementary charge, $\varepsilon_{0}$ the permittivity in vacuum and $\varepsilon_{\mathrm{r}}$ the dielectric constant.

From the experimental data shown in Fig. 3, we obtain a full set of parameters for Eqs. 3 and 4: $\mu_{0}=6.77 \cdot 10^{-5}$ $\mathrm{cm}^{2} / \mathrm{Vs}, \Delta E=0.58 \pm 0.03 \mathrm{eV}, T_{0}=357 \pm 53 \mathrm{~K}$, and $\varepsilon_{\mathrm{r}}=4.0 \pm 0.6$. Blom and Vissenberg found a similar temperature and field dependence of the hole drift mobility in a soluble poly ( $p$-phenylene vinylene) derivative [8] with a zero field mobility and a characteristic energy $\Delta E$ in the same range as we determine in the case of $\mathrm{Alq}_{3}$.

\section{Hole mobility in $\mathbf{A l q}_{3}$}

For the measurement of the hole drift mobility in $\mathrm{Alq}_{3}$, a special DHS device was designed (see Fig. 1b). The upper $\mathrm{Alq}_{3}$ layer serves as electron injection and emission layer. To identify the recombination zone this $\mathrm{Alq}_{3}$ layer is partially doped with $2 \%$ DCM. The $50 \mathrm{~nm}$ thick TPD layer blocks electrons injected at the cathode. The thickness of the $\mathrm{Alq}_{3}$ layer enclosed between two TPD layers was varied from 50 to $200 \mathrm{~nm}$ to verify that the measured delay time is determined by the hole drift mobility in this $\mathrm{Alq}_{3}$ layer. The lower $10 \mathrm{~nm}$ thick TPD layer serves as hole injecting layer.

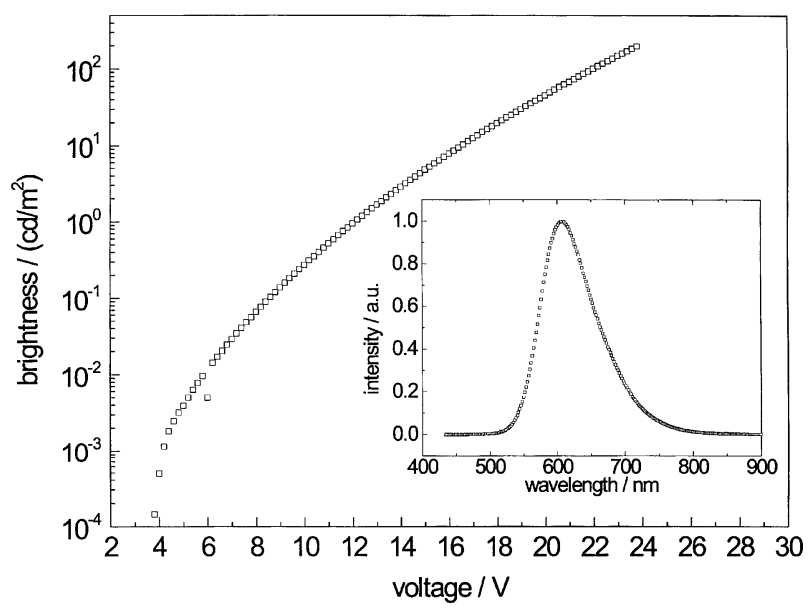

Fig. 4. Electroluminescence characteristic of the DHS device with a 50 $\mathrm{nm} \mathrm{Alq}{ }_{3}$ layer sandwiched between two TPD layers as shown in Fig. 1. The inset shows the emission spectrum of the DHS sample.

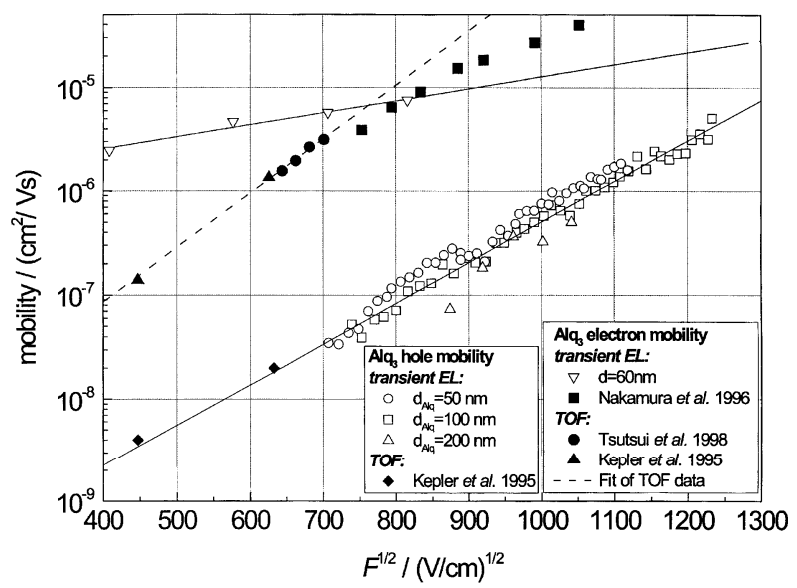

Fig. 5. Field dependence of charge carrier drift mobilities in $\mathrm{Alq}_{3}$ at 300 $\mathrm{K}$ and comparison to TOF data by Kepler et al. [2] and Tsutsui et al. [9] or data of transient EL measurements by Nakamura et al. [4].

As measurements of electroluminescence-voltage characteristics show EL intensity at voltages higher than approximately $4 \mathrm{~V}$ (see Fig. 4), one has to conclude that either holes migrate through the $\mathrm{Alq}_{3}$ sandwiched between two TPD layers or electrons drift through the $50 \mathrm{~nm}$ thick TPD layer. However, the emission spectrum (see inset in Fig. 4) gives unique evidence that charge carrier recombination occurs only in the DCM doped $\mathrm{Alq}_{3}$ layer because no EL intensity was measured below wavelengths of $520 \mathrm{~nm}$ where one would expect the maximum of $\mathrm{Alq}_{3}$ emission.

From TOF measurements, the drift mobilities of holes in TPD and electrons in $\mathrm{Alq}_{3}$ are supposed to be higher by orders of magnitude than the hole drift mobility in $\mathrm{Alq}_{3}$. For this reason, the transit time of electrons in the upper $\mathrm{Alq}_{3}$ layer and the transit time of the holes through both TPD layers can be neglected. The delay times observed for the DHS device are longer by more than one order of magnitude as compared to the SHS device. The assumption that these long transit times originate from the lower hole drift mobility in $\mathrm{Alq}_{3}$ can be verified by measurements on devices with different thickness of the $\mathrm{Alq}_{3}$ layer sandwiched between the two TPD layers, since the data for all three thicknesses fall on the same line (see Fig. 5).

For the calculation of the electric field within the $\mathrm{Alq}_{3}$ layer sandwiched between the two TPD layers, we assume the applied voltage to drop homogeneously over this $\mathrm{Alq}_{3}$ layer and the upper $50 \mathrm{~nm}$ thick TPD layer. With the thickness of this $\mathrm{Alq}_{3}$ layer varied from 50 to $200 \mathrm{~nm}$ and the upper TPD layer of thickness $50 \mathrm{~nm}$, we obtain an electric field of

$F=\left(V-V_{\mathrm{bi}}\right) /\left(d_{\mathrm{Alq}_{3}}+d_{\mathrm{TPD}}\right)$.

With respect to the higher drift mobilities of electrons in $\mathrm{Alq}_{3}$ and holes in TPD this estimation is reasonable as the time for the injected charge carriers to traverse the first organic layer can be neglected. As a consequence, the 
space charges within both organic layers next to the electrodes are built up immediately leading to a negligible voltage drop over these layers.

Then the hole drift mobility in $\mathrm{Alq}_{3}$ can be calculated as

$\mu_{\mathrm{h}, \mathrm{Alq}_{3}}=d_{\mathrm{Alq}_{3}} /\left(t_{\mathrm{d}} \cdot F\right)$.

Fig. 5 shows the hole drift mobility in $\mathrm{Alq}_{3}$ measured at a temperature of $300 \mathrm{~K}$ using DHS devices with $d_{\mathrm{Alq}_{3}}=50$, $100,200 \mathrm{~nm}$. From the linear fit in the Poole-Frenkel plot, we obtain a dependence of the hole drift mobility on the electric field according to

$$
\begin{aligned}
\mu_{\mathrm{h}, \mathrm{Alq}_{3}}= & (6 \pm 1) 10^{-11} \mathrm{~cm}^{2} / \mathrm{Vs} \\
& \times \exp \left[\sqrt{F} \times(9.0 \pm 0.2) 10^{-3} \sqrt{\mathrm{cm} / \mathrm{V}}\right] .
\end{aligned}
$$

We find good agreement with results of Kepler et al. [2] who measured field dependent hole drift mobility in thick $\mathrm{Alq}_{3}$ films using the TOF method.

\section{Discussion}

Fig. 5 also shows the field dependence of the electron mobility at room temperature obtained by TOF experiments and transient electroluminescence. Compared to TOF measurements by Kepler et al. [2] and Tsutsui et al. [9] or transient EL measurements by Nakamura et al. [4], our data of the electron drift mobility in $\mathrm{Alq}_{3}$ show a higher zero field mobility and a weaker field dependence. A possible reason for the deviations of TOF data and transient EL measurements may be found in the different sample thickness used for TOF and transient EL, since for dispersive transport, the effective mobilities are often observed to be thickness dependent. On the other hand, the determination of electron mobilities from transient electroluminescence on multilayer devices also relies on certain assumptions mentioned above, which need not be fulfilled equally well in different devices. In the calculation of the electron drift mobility from transient electroluminescence by Nakamura et al., a homogeneous voltage drop over all organic layers and no built-in potential was assumed. However, if the latter is considered and the voltage drop over the hole transporting layers is neglected (as discussed above) the data by Nakamura et al. yield a similar field dependence and slightly lower absolute values of the electron mobility as compared to our data.

The comparison of the electron and hole drift mobility in $\mathrm{Alq}_{3}$ at $300 \mathrm{~K}$ obtained from transient electroluminescence shows that the hole mobility at low fields is lower by almost three orders of magnitude. However, with increasing electric field, the difference of the mobilities of both charge carrier types becomes smaller due to the stronger dependence of the hole drift mobility on the electric field. From this point of view, the hole transport in $\mathrm{Alq}_{3}$ cannot be neglected generally in organic LEDs.

\section{Acknowledgements}

Financial support by the Bayerische Forschungsstiftung, the Volkswagen Stiftung and the Sonderforschungsbereich 481 is gratefully acknowledged.

\section{References}

[1] C.W. Tang, S.A. VanSlyke, Appl. Phys. Lett. 51 (1987) 913.

[2] R.G. Kepler, P.M. Beeson, S.J. Jacobs, R.A. Anderson, M.B. Sinclair, V.S. Valencia, P.A. Cahill, Appl. Phys. Lett. 66 (1995) 3618.

[3] L.-B. Lin, S.A. Jenekhe, R.H. Young, P.M. Borsenberger, Appl. Phys. Lett. 70 (1997) 2052.

[4] H. Nakamura, C. Hosokawa, T. Kusumoto, Inorganic and Organic Electroluminescence, in: R.H. Mauch, H.-E. Gumlich (Eds.), Wissenschaft Technik Verlag, Berlin, 1996, p. 95.

[5] V.R. Nikitenko, Y.-H. Tak, H. Bässler, J. Appl. Phys. 84 (1998) 2334.

[6] J. Frenkel, Phys. Rev. 54 (1938) 647.

[7] W.D. Gill, J. Appl. Phys. 43 (1972) 5033.

[8] P.W.M. Blom, M.C.J.M. Vissenberg, Phys. Rev. Lett. 80 (3819) (1998).

[9] T. Tsutsui, H. Tokuhisa, M. Era, Polymer Photonic Devices, SPIE Proc. 3281 (1998) 230. 